AperTO - Archivio Istituzionale Open Access dell'Università di Torino

Cytoarchitectural analysis of the neuron-to-glia association in the dorsal root ganglia of normal and diabetic mice

This is a pre print version of the following article:

Original Citation:

Availability:

This version is available http://hdl.handle.net/2318/1759923

since $2020-10-27 T 18: 51: 22 Z$

Published version:

DOI:10.1111/joa.13252

Terms of use:

Open Access

Anyone can freely access the full text of works made available as "Open Access". Works made available under a Creative Commons license can be used according to the terms and conditions of said license. Use of all other works requires consent of the right holder (author or publisher) if not exempted from copyright protection by the applicable law. 


\section{IIIS AperTO}

UNIVERSITÀ

DEGLI STUDI

DI TORINO

This is the author's final version of the contribution published as:

Elisa Ciglieri ${ }^{1^{\circ}}$, Maurizia Vacca ${ }^{2}$, Francesco Ferrini ${ }^{1, \#}$, Mona A. Atteya ${ }^{3}$, Patrizia Aimar ${ }^{1}$, Elisa Ficarra ${ }^{2}$, Santa Di Cataldo ${ }^{2}$, Adalberto Merighi $^{1 \S}$, Chiara Salio ${ }^{1 *}$

Cytoarchitectural analysis of the neuron-to-glia association in the dorsal root ganglia of normal and diabetic mice. Journal of Anatomy, 237 (5):988-997. 2020

DOI:10.1111/joa.13252

The publisher's version is available at:

https://onlinelibrary.wiley.com/doi/10.1111/joa.13252

When citing, please refer to the published version.

Link to this full text: 


\title{
Cytoarchitectural analysis of the neuron-to-glia association in the dorsal root ganglia of normal and diabetic mice.
}

\author{
Running title: Neuron-glia spatial relationship in DRGs \\ Elisa Ciglieri ${ }^{1}{ }^{\circ}$, Maurizia Vacca ${ }^{2}$, Francesco Ferrini1,\#, Mona A. Atteya ${ }^{3}$, Patrizia \\ Aimar ${ }^{1}$, Elisa Ficarra ${ }^{2}$, Santa Di Cataldo ${ }^{2}$, Adalberto Merighi ${ }^{1 \S}$, Chiara Salio ${ }^{1 *}$. \\ ${ }^{1}$ Department of Veterinary Sciences, University of Turin, Grugliasco, Italy. \\ - Max Planck Institute for Metabolism Research, Cologne, Germany. \\ \# Department of Psychiatry \& Neuroscience, Université Laval, Québec, QC, Canada \\ $\S$ National Institute of Neuroscience, Grugliasco, Italy. \\ 2 Department of Control and Computer Engineering, Politecnico di Torino, \\ Italy. \\ ${ }^{3}$ Department of Biochemistry, Faculty of Science, Alexandria University, Egypt.
}

${ }^{*}$ Correspondence:

Dr. Chiara Salio

Department of Veterinary Sciences

University of Turin

Largo Braccini, 2

10095 Grugliasco, Italy

chiara.salio@unito.it

\begin{abstract}
Dorsal root ganglia (DRGs) host the somata of sensory neurons which convey information from the periphery to the central nervous system. These neurons have heterogeneous size and neurochemistry, and those of small-to-medium size, which play an important role in nociception, form two distinct subpopulations based on the presence (peptidergic) or absence (non-peptidergic) of transmitter neuropeptides. Few investigations have so far addressed the spatial relationship between neurochemically different subpopulations of DRG neurons and glia. We used a whole-mount mouse lumbar DRG preparation, confocal microscopy and computer-aided 3D analysis, to unveil that IB4+ non-peptidergic neurons form small clusters of $4.7 \pm 0.26$ cells, differently from CGRP+ peptidergic neurons that are, for the most, isolated (1.89 \pm 0.11 cells). Both subpopulations of neurons are ensheathed by a thin layer of satellite glial cells (SGCs) that can be observed after immunolabeling with the specific marker glutamine synthetase (GS). Notably, at the ultrastructural level we observed that this glial
\end{abstract}


layer was discontinuous, as there were patches of direct contact between the membranes of two adjacent IB4+ neurons.

To test whether this cytoarchitectonic organization was modified in the diabetic neuropathy, one of the most devastating sensory pathologies, mice were made diabetic by streptozotocin (STZ). In diabetic animals, cluster organization of the IB4+ non-peptidergic neurons was maintained, but the neuro-glial relationship was altered, as STZ treatment caused a statistically significant increase of GS staining around CGRP+ neurons but a reduction around IB4+ neurons. Ultrastructural analysis unveiled that SGC coverage was increased at the interface between IB4+ cluster-forming neurons in diabetic mice, with a 50\% reduction in the points of direct contacts between cells. These observations demonstrate the existence of a structural plasticity of the DRG cytoarchitecture in response to STZ.

\section{Keywords}

Dorsal Root Ganglia; 3D computer-aided reconstruction; Satellite Glial Cells; Diabetic Peripheral Neuropathy; Peptidergic neurons; Non-peptidergic neurons.

\section{Introduction}

Except for certain specialized receptors, the cell bodies of the primary sensory neurons are grouped in a series of ganglia of the peripheral nervous system associated to the brain or the spinal cord. In the latter they are situated along the dorsal roots of the spinal nerves and commonly referred to as dorsal root ganglia (DRGs). DRGs are made of pseudounipolar neurons and the surrounding glia. Neurons may be simply classified into small-to-medium sized cells, mostly specialized in encoding noxious stimuli, and medium-to-large sized cells, typically encoding innocuous low-threshold stimuli (Lawson \& Waddell, 1991; Lawson, 2002). However, these two populations are highly heterogeneous, and small-to-medium sized DRG neurons may be further subdivided into peptidergic or non-peptidergic cells. Peptidergic neurons are about $30-45 \%$ of the total number of DRG neurons, and typically express one or more neuropeptides among which the more common is the calcitonin gene-related peptide (CGRP) (Gibson et al., 1984; Lawson, 1995), which is now accepted as the best marker to identify these cells. Non-peptidergic neurons, representing another third of the total population of DRG neurons, are of smaller size and, at least in mouse, can be specifically identified after histochemical labeling with the isolectin B4 (IB4) from Griffonia simplicifolia (Silverman and Kruger, 1990).

Irrespective of their neurochemical heterogeneity, neurons are generally believed to be evenly distributed within ganglia, and only few studies have probed this view by appropriate anatomical investigations (Burton \& McFarlane, 1973; Wessels et al., 1990; PuigdellívolSánchez et al., 1998; Yan et al., 2002; Ostrowski et al., 2017). Again disregarding their heterogeneity, the cell bodies of the DRG neurons are individually enwrapped by satellite glial cells (SGCs), so that each neuron forms a discrete unit, sharply separated anatomically and insulated electrically from the adjacent nerve cells (Pannese, 2010).

Still, the neurochemical diversity of the DRG neurons is very important, because it underscores their functional diversification, particularly regarding the intervention in the 
initial processing of nociceptive stimuli (see Merighi, 2018). Remarkably, whereas many studies have demonstrated that SGCs go through important functional alterations in pain, specifically in the diabetic neuropathy (Hanani et al., 2014; Verkhratsky \& Fernyhough, 2014), as well as in the presence of sustained visceral pain (Huang et al., 2010), no data are available as regarding the possibility that the DRG neurons undergo structural plasticity when pain processing is altered or disturbed.

To provide further information about the neuron-to-glia structural association in mouse DRGs, as well as on its putative plasticity in the diabetic neuropathy, we here used confocal microscopy coupled with 3D computer-aided analysis in a whole-mount ganglion preparation (Ciglieri et al., 2016) and transmission electron microscopy (TEM) to study the tridimensional organization of CGRP+ and IB4+ DRG neurons, as well as their SGCs in normal and diabetic mice. Diabetes was induced by a single high dose injection of streptozotocin (STZ). STZ has a structural similarity with glucose and is taken up by pancreatic $\beta$ cells via glucose transporter 2 , causing the death of the cells by DNA fragmentation and impairment of glucose transport (Ventura-Sobrevilla et al., 2011). This model induces a severe and long lasting neuropathy, characterized by variable alterations of sensory profiles (Ventura-Sobrevilla et al., 2011).

Together, our results showed that the IB4+ non-peptidergic neurons were organized in small clusters, while the CGRP+ peptidergic neurons were evenly scattered across the DRGs. While this organization remained unaltered in diabetic mice, the SGCs surrounding the two populations of DRG neurons were subjected to extensive structural alterations that may be a histological substrate at the basis of nociceptive alterations in diabetes.

\section{Methods}

\section{Animals}

All experimental procedures were approved by the Italian Ministry of Health and the Committee of Bioethics and Animal Welfare of the University of Torino (417/2016-PR). Animals were maintained according to the NIH Guide for the Care and Use of Laboratory Animals and to current EU and Italian regulations.

Male CD1 mice (20-30 g) were housed in a controlled environment and maintained on a 12/12-hour light/dark cycle with food and water ad libitum. All experiments were performed in both control (normoglycemic) and diabetic (hyperglycemic) mice. To induce diabetes, animals at postnatal day 30 (P30) received one single intraperitoneal injection of streptozotocin (STZ - Sigma, St. Louis, MO, USA, Cat\# S0130). STZ was administered at a dose of $150 \mathrm{mg} / \mathrm{kg}$ freshly dissolved in $0.1 \mathrm{M}$ citrate buffer $\mathrm{pH} 4.5$ to experimental animals, whereas control mice only received the vehicle. Four weeks later (P60), following tail venipuncture in 5 hour-fasted animals, glycemia was measured using a glucose oxidase impregnated test strip (Glucocard sensor; Menarini, Firenze, Italy). Only mice with a blood glucose concentration higher than $300 \mathrm{mg} / \mathrm{dL}$ were considered diabetic and used for the subsequent experiments (see Fig. S1).

\section{Whole-mount DRG preparation}

Immunofluorescence experiments were performed on a whole-mount lumbar DRG preparation, as previously described (Ciglieri et al., 2016). Briefly, mice (control $\mathrm{N}=41$, diabetic $\mathrm{N}=31$ ) were anesthetized with a lethal dose of sodium pentobarbital $(30 \mathrm{mg} / \mathrm{kg}$, intraperitoneal). Dissection of the lumbar DRGs was then performed by constantly maintaining tissues in an ice-cold cutting solution, containing: sucrose $252 \mathrm{mM}, \mathrm{KCl} 2.5 \mathrm{mM}$, $\mathrm{NaHCO}_{3} 26 \mathrm{mM}, \mathrm{NaH}_{2} \mathrm{PO}_{4} 1.25 \mathrm{mM}$, D-glucose $10 \mathrm{mM}$, kynurenate $1 \mathrm{mM}, \mathrm{MgCl}_{2} 3 \mathrm{mM}, \mathrm{CaCl}_{2}$ $1.5 \mathrm{mM}$, saturated with $95 \% \mathrm{O}_{2}-5 \% \mathrm{CO}_{2}$. DRGs were removed after cutting the vertebral column along the midline; then, they were incubated for 1 hour at $37^{\circ} \mathrm{C}$ in constantly oxygenated artificial cerebro-spinal fluid (aCSF), containing: $\mathrm{NaCl} 126 \mathrm{mM}, \mathrm{KCl} 2.5 \mathrm{mM}, \mathrm{D}-$ glucose $10 \mathrm{mM}, \mathrm{NaHCO}_{3} 26 \mathrm{mM}, \mathrm{NaH}_{2} \mathrm{PO}_{4} 1.25 \mathrm{mM}, \mathrm{CaCl}_{2} 2 \mathrm{mM}, \mathrm{MgCl}_{2} 1.5 \mathrm{mM}$ and collagenase 
(7 mg/mL, collagenase type 3; Worthington, NJ, USA, Cat\# LS004180) to digest the outer connective capsule of the ganglia and to allow for better penetration of the immunoreactants for 3D analysis.

\section{Immunofluorescence}

Acutely dissected, collagenase-treated DRGs were fixed for $30 \mathrm{~min}$ with $4 \%$ paraformaldehyde in phosphate buffer (PB; $0.1 \mathrm{M}, \mathrm{pH} 7.4$ ), washed several times in phosphate buffered saline (PBS; $0.02 \mathrm{M}, \mathrm{pH}$ 7.4), and then processed for immunofluorescence as follows:

$i$ - They were pre-incubated in PBS containing 6\% bovine serum albumin for $1 \mathrm{~h}$, followed by overnight incubation at $4^{\circ} \mathrm{C}$ with an IB4 biotin-conjugate (1:250; Sigma, Cat\# L2140), washed in PBS and incubated for $1 \mathrm{~h}$ with Extravidin-FITC (1:500; Sigma, Cat\# E276);

$i i$ - They were pre-incubated in $1 \%$ normal goat serum and $0.1 \%$ Triton $\mathrm{X}-100$ for $1 \mathrm{~h}$, and then incubated overnight at $4{ }^{\circ} \mathrm{C}$ with the following primary antibodies: polyclonal rabbit anti-CGRP antibody (1:500; Sigma, Cat\# C8198; Salio \& Ferrini, 2016); monoclonal mouse anti-glutamine synthetase (GS) antibody, clone GS-6 which specifically stains SGCs (1:50; Merck, Cat\# MAB302; Magni et al., 2015; Rajasekhar et al., 2015). After washing in PBS, DRGs were incubated for $1 \mathrm{~h}$ with appropriate secondary antibodies (1:1000; anti-rabbit Alexa Fluor 633Cat\# A-21070, anti-rabbit Alexa Fluor 594-Cat\# A-11012, anti-mouse Alexa Fluor 546-Cat\# A-11003; Thermo Fisher, Waltham, MA, USA).

Negative controls performed by omitting the primary antibodies completely abolished the specific staining.

In a subset of experiments, ganglia were stained with 4',6-diamidino-2-phenylindole dihydrochloride (DAPI; Sigma, Cat\# D9542) by a pre-incubation in PBS containing 0.1\% Triton X-100 for $30 \mathrm{~min}$ followed by $15 \mathrm{~min}$ in $300 \mathrm{nM}$ DAPI.

To obtain Z-series reconstructions, immunostained DRGs were transferred on slides modified ad hoc to maintain their 3D volume (Ciglieri et al., 2016) and mounted with Vectashield medium (Vector Labs, Burlingame, CA, USA, Cat\# H-1000). Immunofluorescence was acquired using a confocal microscope (TCS SP5; Leica Microsystems, Wetzlar, Germany) with a 20x objective (N.A. 0.17). DAPI was excited with a $405 \mathrm{~nm}$ diode laser, FITC with a $488 \mathrm{~nm}$ argon laser, Alexa Fluor 546 and 594 with a 547 nm HeNe laser and Alexa Fluor 633 with a 633 nm HeNe laser. Pinhole was kept at 1 airy unit. Gain and offset were initially set for each fluorophore and maintained constant in the subsequent acquisitions. Confocal optical sections were taken at $3.5 \mu \mathrm{m}$ intervals along the $\mathrm{Z}$ axis in sequential mode.

\section{Electron microscopy}

Eighteen DRGs (3 DRGs/mouse from three control and three diabetic mice) dissected out from mice euthanized as previously described, were fixed in $1 \%$ paraformaldehyde $+2 \%$ glutaraldehyde in $\mathrm{PB}(0.1 \mathrm{M}, \mathrm{pH} 7.4)$ overnight at $4^{\circ} \mathrm{C}$. After washing in $\mathrm{PB}$, they were postfixed in osmium ferrocyanide (1 volume of $2 \%$ aqueous osmium tetroxide : 1 volume of $3 \%$ potassium ferrocyanide) for $1 \mathrm{~h}$ at $4^{\circ} \mathrm{C}$, dehydrated for $15 \mathrm{~min}$ in increasing concentrations of acetone $(30 \%, 60 \%, 90 \%, 100 \%)$, progressively infiltrated with Spurr resin (Electron Microscopy Sciences, Hatfield, PA, USA; Cat\#14300; data from manufacturer) and embedded in $0.5 \mathrm{~mL}$ Eppendorf tubes $\left(24 \mathrm{~h}\right.$ at $\left.70^{\circ} \mathrm{C}\right)$.

Ultrathin sections (80 nm thickness) were cut with an ultramicrotome (EM UC6; Leica), collected on uncoated nickel grids (200 mesh) and immunostained following a classical postembedding protocol. Sections were treated for $1 \mathrm{~min}$ with a saturated aqueous solution of 
sodium metaperiodate, rinsed in 1\% Triton X-100 in Tris-buffered saline (TBS; $0.5 \mathrm{M}$ ), and then incubated for $1 \mathrm{~h}$ in $6 \%$ bovine serum albumin in TBS. Grids were then transferred overnight on drops of the IB4 biotin-conjugate (1:20, Sigma, Cat\# L2140). After rinsing in TBS, they were incubated in streptavidin coupled to $20 \mathrm{~nm}$ colloidal gold particles (1:15; BBI Solutions, Crumlin, UK, Cat\# EM.STP20), transferred into drops of $2.5 \%$ glutaraldehyde in cacodylate buffer $0.05 \mathrm{M}$ and, finally, washed in distilled water. Sections were counterstained 10 min with lead citrate before observation with a JEM-1010 transmission electron microscope (Jeol, Tokyo, Japan) equipped with a side-mounted CCD camera (Mega View III, Olympus Soft Imaging System, Munster, Germany).

To assess the neuron-glia distribution in the clusters formed by the IB4+ neurons, quantitative ultrastructural analysis was performed onto sixty randomly selected clusters from control $(n=30)$ and diabetic $(n=30)$ DRGs. To do so, IB4+ clustered neurons were photographed at 15,000x magnification by an operator unaware of the experimental group. Individual micrographs were collated together with Photoshop CS2 9 (Adobe Systems, San Jose, CA, USA) to obtain a single picture of the cluster and then analyzed with the ImageJ Software (NIH, Bethesda, USA). Specifically, the length of the plasma membranes' apposition between two IB4+ clustered neurons was measured, and their distance calculated over 10 equally spaced points. Opposing membranes were considered in "direct contact" when intermembrane distances were $\leq 30-40 \mathrm{~nm}$. This threshold was set assuming that the extracellular space is $\leq 20 \mathrm{~nm}$ and each plasma membrane is about $5 \mathrm{~nm}$ thick (Faisal et al., 2005). A contact index was calculated by dividing the number of intermembrane contact points with distance $\leq 40 \mathrm{~nm}$ by the length of the neuronal interface. Then, the proportion of IB4+ profiles sharing at least one point of contact in control and STZ-treated mice was quantified.

\section{Computerized analysis of neuronal clusterization}

The spatial distribution of neurons in DRGs was analyzed by an in-house developed software for automated 3D analysis (3DRG;see Di Cataldo et al., 2016, Supporting Information Data S1 and Fig. S2). Analysis was performed on confocal images of the immunostained DRGs to detect the peptidergic (CGRP+) and non-peptidergic (IB4+) neuronal populations.

\section{Analysis of SGCs after immunofluorescence staining}

The relationship between SGCs and CGRP+/IB4+ neurons was investigated by 1) counting the SGCs surrounding each neurochemically identified neuron, 2) measuring the fluorescence intensity associated with the SGC marker GS around each identified neuron.

1) The number of SGCs per sensory neuron was estimated by counting the number of DAPI stained nuclei surrounding the equatorial optical section of the neuron, i.e. the largest section on the z-axis. The number of nuclei was normalized to the cross-sectional area to correct for differences in neuronal size.

2) GS fluorescence intensity was measured at the equatorial optical section (see Fig. 3C). To obtain an unbiased estimate of GS distribution around each neuronal cell body, GS fluorescence intensity was measured by the ImageJ Software (https://imagej.nih.gov/ij/) along four lines passing through the optical section center and crossing its membrane at 8 equally spaced points. Since GS staining was concentrated around the neuronal membrane, eight peaks of GS fluorescence were detected. GS fluorescence intensity was measured at each peak (obtained by averaging three consecutive pixels around the peak; pixel size $=1.3 \mu \mathrm{m}$ ) and normalized to the background value (measured at the center of the neuronal cell body). For each identified neuron, the maximum, minimum and mean GS fluorescence was obtained in order to estimate both the fluorescence intensity of SGC marker and its distribution around the sensory neurons (see Fig. 3C). 


\section{Statistics}

Statistical analysis was performed with GraphPad Prism 7. Differences were evaluated by using t-test for independent samples, two-way ANOVA or Mann-Whitney test where appropriate. All data were reported as mean \pm SEM, with $n$ indicating the number of cells. Values of $P<0.05$ were considered statistically significant.

\section{Results}

\section{IB4+ non-peptidergic, but not CGRP+ peptidergic neurons are organized in small clusters}

After analysis with the $3 D R G$ software on 147 DRGs obtained from 29 control and 23 diabetic mice, CGRP+ neurons resulted to be randomly scattered across the entire ganglion volume (Fig. 1A), whereas IB4+ neurons were grouped in clusters (Fig. 1B). Each cluster of IB4+ neurons was composed of a mean of $4.7 \pm 0.26$ cells (Fig. 1C). CGRP+ cells were found in clusters that were made of $1.89 \pm 0.11$ cells. The difference between the number of neurons/cluster between the two subpopulations of DRG cells was statistically significant ( $t$ test, $\mathrm{P}<0.001$; Fig. $1 \mathrm{C}$ ). There were no numerical alterations in the number of cells/cluster when the DRGs from diabetic mice were compared to control mice (Fig. 1C).

\section{The number of SGCs is higher around CGRP+ than IB4+ neurons}

After nuclear staining with DAPI (Fig. 2A-D), the number of SGC nuclei surrounding IB4+ and CGRP+ neurons were calculated and statistically analyzed (Fig. 2E). Artifacts due to the difference in size of the two subpopulations of sensory neurons were minimized by normalizing the number of SGC nuclei to the major cross-sectional area of the neurons themselves. Higher numbers of glial cells nuclei/area were consistently observed around CGRP+ neurons as compared to IB4+ neurons. In controls, SGCs nuclei were $12 \pm 0.4 * 10^{-3} / \mu \mathrm{m}^{2}$ around CGRP+ neurons $(\mathrm{n}=80)$, while they were $7 \pm 0.4^{*} 10^{-3} / \mu \mathrm{m}^{2}$ around IB4+ neurons $(\mathrm{n}=70$; t-test, $\mathrm{P}<0.001)$. Similarly, in diabetic mice SGCs nuclei were $12 \pm 1^{*} 10^{-3} / \mu \mathrm{m}^{2}$ around CGRP+ neurons $(\mathrm{n}=40)$, while they were $7 \pm 1^{*} 10^{-3} / \mu \mathrm{m}^{2}$ around IB4+ neurons $(\mathrm{n}=30 ; \mathrm{t}$-test, $\mathrm{P}<0.001)$. After two-way ANOVA, the differences in the number of SGCs surrounding the two identified populations of nociceptors resulted to depend on the cell phenotype but unaffected by the STZ treatment (two-way ANOVA, effect of treatment: $\mathrm{F}(1,216)=0.02, \mathrm{P}=0.89$; effect of the cell phenotype: $\mathrm{F}(1,216)=84.89, \mathrm{P}<0.001$; treatment factor-interaction between factors: $\mathrm{F}(1$, 216) $=0.48, \mathrm{P}=0.16$ Fig. 2D).

\section{The SGC marker glutamine synthetase (GS) is differentially affected by}

\section{diabetes according to the cell phenotype.}

The distribution of SGCs around CGRP+ and IB4+ sensory neurons was analyzed indirectly, by measuring GS immunofluorescence (Fig. 3A-C).

Under control conditions, mean GS staining was more intense around IB4+ neurons than CGRP+ neurons (Fig. 3D, t-test, $\mathrm{P}=0.03$ ). Conversely, both minimal and mean fluorescence intensities were higher around CGRP+ neurons than IB4+ neurons in diabetic mice (Fig. 3E, $t$ test, $\mathrm{P}=0.02$ and 0.03 ). The two-way ANOVA analysis demonstrated a significant interaction between treatment and cell phenotype per each level of fluorescence intensity analyzed (Fig $3 \mathrm{~F}$, minimal fluorescence, $\mathrm{F}(1,218)=9.49, \mathrm{P}=0.002$; Fig. $3 \mathrm{G}$, mean fluorescence, $\mathrm{F}(1,218)=$ 9.26, $\mathrm{P}=0.003$; Fig. $3 \mathrm{H}$, maximal fluorescence, $\mathrm{F}(1,218)=6.13, \mathrm{P}=0.01)$. Specifically, STZ 
treatment induced a significant decrease of minimal and mean fluorescence intensity around IB4+ neurons (minimal fluorescence intensity, t-test, $\mathrm{P}=0.001$, Fig. 3F; mean fluorescence intensity, t-test, $P=0.005$, Fig. $3 G$ ) and a significant increase of the maximal GS immunofluorescence intensity around CGRP+ neurons ( $t$ test $\mathrm{P}=0.048$, Fig. $3 \mathrm{H}$ ). Altogether, STZ treatment induced an overall reduction of the glial layer around IB4 neurons, as detectable by GS staining, while causing a hypertrophic reaction around CGRP neurons.

\section{Ultrastructural analysis demonstrates a reduction in the juxtaposition of the cell membranes of clustered IB4+ DRG neurons under diabetic conditions}

The presence of glia around the DRG neurons can be easily recognized without specific labels as previously described by Pannese (1981, 2010, Fig. 4A-D). In individual ultrathin sections, IB4+ clusters consisted of two-three cells and the occurrence of clusters was confirmed in both control (Fig. 5A) and diabetic animals (Fig. 5B). In controls, the SGC sheet became progressively thinner at the interface between the IB4+ neurons of the same cluster and, in some points, the membrane of two opposing neurons appeared in direct contact (Fig. 5C). After quantitative analysis, the contact index between cluster-forming IB4+ neurons was markedly reduced in diabetic animals (Mann-Whitney test, $\mathrm{P}<0.01$, Fig. 5D, E). Similarly, the proportion of neuronal interface exhibiting at least one point of direct contact was reduced of about $50 \%$ in these mice (Fisher exact test, $\mathrm{P}<0.05$, Fig. 5F).

Results are graphically summarized in Figure 6.

\section{Discussion}

In the present study, we found that the non-peptidergic IB4+ neurons in DRGs form small clusters, differently from the CGRP+ peptidergic neurons. This configuration is unaltered in diabetic mice. However, hyperglycemic conditions deeply affect the neuron-glia structural relationship between cluster-forming neurons, thus suggesting that the 3D organization of these cells has a functional impact.

\section{Structural relationship between sensory neurons and SGCs in DRGs}

It is widely accepted that there are no chemical synapses between the DRG neurons. Yet several forms of neuron-to-neuron and neuron-to-glia communication occur in DRGs particularly under conditions of inflammation and/or pain. Thus, electrical synapses (gap junctions) between DRG neurons are rare under basal conditions (Ledda et al 2009), but their number may increase together with neuron-to-neuron dye coupling in experimental inflammation (Ledda et al., 2009; Huang et al. 2010). Noteworthy, it was also demonstrated that coupled activation of DRG neurons was mediated by an injury-induced upregulation of gap junctions in SGCs and that neuronal coupling contributed to pain hypersensitivity (Kim et al., 2016). Then, very recently, the gas messenger nitric oxide (NO) released by the DRG neurons was shown to induce activation of SGCs and to increase gap-junctional communication in vitro (Belzer and Hanani, 2019). Therefore, despite that adult DRG neurons are insulated by a non-conductive glial layer, which minimizes their direct interactions (Ohara et al., 2009), SGCs intervene in regulating neuronal excitability in DRGs. Mono/bidirectional gap junction-mediated neurotransmission between the DRG neurons and the SGCs may not be the only type of communication between these cells. Namely, other authors have described the occurrence of "sandwich synapses" between the DRG neurons and glia (Rozanski et al., 2013). Structurally, the sandwich synapses described by Rozanski and colleagues consist of neuron-glial cell-neuron trimers, in which membranes are closely apposed in the absence of any ultrastructural differentiation if not a narrowing of intermembrane clefts. These authors 
have shown an unidirectional ionic current following through sandwich synapses whereby a DRG "cis" neuron forms a first synapse with the SGC that, in turn, forms a second synapse with an adjacent "trans" neuron (see Figure 6 in Rozanski et al., 2013). According to the original sandwich synapse hypothesis, stimulation in a given "cis" neuron propagates to neighboring nerve cells following the activation of the purinergic P2Y12 receptors expressed by the surrounding SGCs, which have been hypothesized to release glutamate that acts onto the NMDA receptors expressed at the membrane of the "trans" neuron (Rozanski et al., 2013). In line with such a possibility, astrocytic glutamate was shown to evoke NMDA receptormediated slow depolarizing inward currents in neurons (Gomez-Gonzalo et al., 2018). In DRGs, the spread of excitation driven through sandwich synapses is enhanced in a variety of pathological pain conditions (Ohara et al., 2009; Wu et al., 2012; Kim et al., 2016). These observations highlight the importance of the spatial contacts among the DRG neurons and between them and the SGCs to support their electrical coupling. In line with this, we here have demonstrated that the IB4+ non-peptidergic nociceptors were organized in small clusters, differently from their CGRP+ peptidergic counterpart. Interestingly, these two subpopulations of DRG neurons also displayed a different association with their surrounding glia. Specifically, IB4+ neurons exhibited a lower number of associated SGCs, and their membranes were directly juxtaposed within the clusters at TEM observation. That glial coverage was incomplete led us to speculate that direct neuron-to-neuron communication could occur. Direct neuronal appositions, in the absence of synaptic specializations, were previously observed between neurochemically unclassified sensory neurons of several species, including lizards (Pannese, 2010), chicks (Rozanski et al., 2012), rats (Pannese, 2010) and rabbits (Khan et al., 2009). Such a structural arrangement is consistent with their functional coupling by mechanisms others than electrical (gap junctions) or sandwich synapses. In their seminal study, Devor \& Wall (1990) were the first to find that about 5\% of DRG neurons induced subthreshold activity in the neighboring nerve cells. Later, slow chemical transmission between DRG neurons somata was demonstrated to take place with the intervention of ATP as a neurotransmitter in chick DRG neurons (Rozanski et al., 2012). In line with these observations, we here identify the contact points between IB4+ neurons as a structural substrate for electrotonic neuronal coupling.

\section{STZ-induced alterations in the neuron to glia association}

Alterations in SGCs function and in their anatomical relationship with sensory neurons strongly affect the spread of excitability across DRGs. SGCs were reported to undergo important changes in their morphology and activity, often described as an activated state, that contributes to pathological pain and favors pathological pain behavior (Hanani, 2012). In addition, direct coupling among clusters of 2-5 DRG neurons, particularly the smaller ones $(<20 \mu \mathrm{m})$, was elegantly demonstrated by in vivo calcium imaging in mice with pathological pain (Kim et al., 2016). SGCs activation also occurred in animals where the diabetic neuropathy was experimentally induced (Hanani et al., 2014; Jia et al., 2018). As early as two weeks after STZ injection, Hanani and coll. (2014) found a significant increase in the expression of the glial fibrillary acidic protein (GFAP) in mouse and rat activated SGCs. SGC activation was also characterized by an increased expression of P2Y12 receptors, which participate to the sandwich synapse mechanism, and connexin 43 , which promotes transglial spread of excitation through the gap junctions (Jia et al., 2018).

Very recently Jia and coll. (2018) found that, in diabetic animals, SGCs activation was preferentially detected at the level of the CGRP-expressing neurons. Our confocal data from STZ-induced diabetic mice support these observations at CGRP neurons; contrariwise, we found a decrease of GS staining around IB4+. However, our ultrastructural study unveiled that 
this reduction in GS staining was not associated to a reduction of glia ensheathment, which increased at interface between IB4+ cluster-forming neurons.

This apparently contradictory finding indicates that the mere immunocytochemical analysis of glia at the confocal microscopy may not be sensitive enough to detect changes in the SGC morphology at nanometric scale. Indeed, within neuronal clusters, glial processes separated the neuronal membranes of few tens of nanometers (Faisal et al., 2005) whose fine alterations may unlikely to be detected at the light microscopy level. Our data indicate that diabetes induced an overall increase in glial coverage at both peptidergic and non-peptidergic neurons in DRGs: at the micrometric level in the former and at the nanometric level in the latter. At neuronal cluster level, diabetes induces a shift from a condition in which IB4+ neurons share extensive contact areas, to a condition in which interneuronal responses might be mediated by the interposing SGCs. Importantly, none of the observed changes in glia distribution around sensory neurons can be explained in terms of change in SGC number, as we were unable to demonstrate any significant change in the number of SGC nuclei in diabetic mice. An interesting result of our study is that we have also observed the presence of some gap junctions between the DRG neurons and the SGCs in both normal and diabetic DRGs. Several papers (quoted above) have in fact demonstrated that neuro-glia gap junctions were the structural and functional substrate to explain the activation of the SGCs that occurs in diabetes, inflammation, or pathological pain.

\section{Acknowledgments}

This work was funded by Fondazione CRT (grant N. RF= 2015.1690 to FF) and Compagnia San Paolo (Fondi di Ateneo 2012 to CS).

\section{Conflict of interest}

The authors declare no conflicts of interest.

\section{Author contributions}

E.C., F.F and C.S. conceived the experiments and participated in their design; E.C. performed immunofluorescence and data acquisition; E.C., M.A.A. and F.F. performed data analysis;: M.V; E.F. and S.D.C developed the Software and 3D-analysis; P.A. and C.S. performed electron microscopy; E.C., F.F., A.M. and C.S contributed to data interpretation; F.F., E.F.; S.D.C.; C.S and A.M. revised, formulated and finalized the submitted manuscript.

\section{References}

Belzer V, Hanani M (2019) Nitric oxide as a messenger between neurons and satellite glial cells in dorsal root ganglia. Glia 67, 1296-1307.

Burton H, McFarlane JJ (1973) The organization of the seventh lumbar spinal ganglion of the cat. J Comp Neurol 149, 215-231.

Ciglieri, E., Ferrini, F., Boggio, E., et al. (2016) An improved method for in vitro morphofunctional analysis of mouse dorsal root ganglia. Annals Anat 207, 62-67.

Di Cataldo S, Tonti S, Ciglieri E, et al. (2016) Automated 3D immunofluorescence analysis of Dorsal Root Ganglia for the investigation of neural circuit alterations: a preliminary study. Annals of Computer Science and Information Systems 9, 65-70. 
Devor M, Wall PD (1990) Cross-excitation in dorsal root ganglia of nerve-injured and intact rats. J Neurophysiol 64, 1733-1746.

Faisal AA, White JA, Laughlin SB (2005) Ion-channel noise places limits on the miniaturization of the brain's wiring. Curr Biol 15, 1143-1149.

Gibson SJ, Polak JM, Bloom SR, et al. (1984) Calcitonin gene-related peptide immunoreactivity in the spinal cord of man and of eight other species. J Neurosci 4 , 3101-3111.

Gómez-Gonzalo M, Zehnder T, Requie LM et al. (2018) Insights Into the Release Mechanism of Astrocytic Glutamate Evoking in Neurons NMDA Receptor-Mediated Slow Depolarizing Inward Curr Glia 66, 2188-2199.

Hanani M, Blum E, Liu S, et al. (2014) Satellite glial cells in dorsal root ganglia are activated in streptozotocin-treated rodents. J Cell and Mol Med 18, 2367-2371.

Hanani M (2012) Intercellular communication in sensory ganglia by purinergic receptors and gap junctions: implications for chronic pain. Brain Res 1487, 183-191.

Huang T-Y, Belzer V, Hanani M (2010) Gap junctions in dorsal root ganglia: possible contribution to visceral pain. J Physiol 14, 647-660.

Jia T, Rao J, Zou L, et al. (2018) Nanoparticle-Encapsulated Curcumin Inhibits Diabetic Neuropathic Pain Involving the P2Y12 Receptor in the Dorsal Root Ganglia. Front Neurosci, 11, 755-767.

Khan AA, Dilkash MNA, Khan MA, et al. (2009) Morphologically atypical cervical dorsal root ganglion neurons in adult rabbit. Biomed Res 20, 45-49.

Kim YS, Anderson M, Park K, et al. (2016) Coupled Activation of Primary Sensory Neurons Contributes to Chronic Pain. Neuron 91, 1085-1096.

Lawson SN (1995) Neuropeptides in morphologically and functionally identified primary afferent neurons in dorsal root ganglia: substance P, CGRP and somatostatin. Prog in Brain Res 104, 161-173.

Lawson SN (2002) Phenotype and function of somatic primary afferent nociceptive neurones with C-, Adelta- or Aalpha/beta-fibres. Exp Physiol 87, 239-244.

Lawson SN, Waddell PJ (1991) Soma neurofilament immunoreactivity is related to cell size and fibre conduction velocity in rat primary sensory neurons. J Physiol, 435, 41-63.

Ledda M, Blum E, De Palo S et al. (2009) Augmentation in gap junction-mediated cell coupling

in dorsal root ganglia following sciatic nerve neuritis in the mouse. Neurosci 164, 15381545.

Magni G, Merli D, Verderio C, et al. (2015) P2Y2 receptor antagonists as anti-allodynic agents in acute and sub-chronic trigeminal sensitization: role of satellite glial cells. Glia, 63, 1256-1269.

Merighi A (2018) Costorage of High Molecular Weight Neurotransmitters in Large Dense Core Vesicles of Mammalian Neurons. Front Cell Neurosci 21, 12:272.

Ohara PT, Vit J-P, Bhargava A, et al. (2009) Gliopathic pain: when satellite glial cells go bad. Neuroscientist 15, 450-463.

Ostrowski AK, Sperry ZJ, Kulik G, et al. (2017) Quantitative models of feline lumbosacral dorsal root ganglia neuronal cell density. J Neurosci Methods 290, 116-124.

Pannese E (1981) The satellite cells of the sensory ganglia. Adv Anat Embryol Cell Biol 65, 1111. 
Pannese E (2010) The structure of the perineuronal sheath of satellite glial cells (SGCs) in sensory ganglia. Neuron Glia Biol 6, 3-10.

Puigdellívol-Sánchez A, Prats-Galino A, Ruano-Gil D, et al. (1998) Sciatic and femoral nerve sensory neurones occupy different regions of the L4 dorsal root ganglion in the adult rat. Neurosci Lett 251, 169-172.

Rajasekhar P, Poole DP, Liedtke W, et al. (2015) P2Y1 Receptor Activation of the TRPV4 Ion Channel Enhances Purinergic Signaling in Satellite Glial Cells. J Biol Chem 290, 2905129062.

Rozanski GM, Kim H, Li Q et al. (2012) Slow chemical transmission between dorsal root ganglion neuron somata. Eur J Neurosci 36, 3314-3321.

Rozanski GM, Li Q, Stanley EF (2013) Transglial transmission at the dorsal root ganglion sandwich synapse: glial cell to postsynaptic neuron communication. Eur J Neurosci 37, 12211228.

Salio C, Ferrini F (2016) BDNF and GDNF expression in discrete populations of nociceptors. Ann Anat 207, 55-61.

Silverman JD, Kruger L (1990) Selective neuronal glycoconjugate expression in sensory and autonomic ganglia: relation of lectin reactivity to peptide and enzyme markers. $J$ Neurocytol 19, 789-801.

Ventura-Sobrevilla J, Boone D, Aguilar, C, et al. (2011). Effect of Varying Dose and Administration of Streptozotocin on Blood Sugar in Male CD1 Mice. Proc. West. Pharmacol. Soc. 54, 5-9.

Verkhratsky A, Fernyhough P (2014) Calcium signalling in sensory neurones and peripheral glia in the context of diabetic neuropathies. Cell Calcium 56, 362-371.

Wessels WJ, Feirabend HK, Marani E (1990) Evidence for a rostrocaudal organization in dorsal root ganglia during development as demonstrated by intra-uterine WGA-HRP injections into the hindlimb of rat fetuses. Brain Res Dev Brain Res 54, 273-281.

Wu A, Green CR, Rupenthal ID, et al. (2012) Role of gap junctions in chronic pain. J Neurosci Res 90, 337-345.

Yan J, Tian R, Horiguchi M (2002) Distribution of sensory neurons of ventral and dorsal cervical cutaneous nerves in dorsal root ganglia of adult rat--a double-label study using DiO and DiI. Okajimas Folia Anat Jpn 79, 129-133.

\section{Figure legends}

Fig. 1 Cluster analysis of CGRP+ and IB4+ neurons in DRGs from control and diabetic mice. Representative pictures of CGRP+ (A) and IB4+ (B) neurons in DRGs from CTR. The enlargements below illustrate the diameters of representative CGRP+ and IB4+ cells. (C) Histogram showing the number of cells per cluster of CGRP+ and IB4+ neurons in vehicletreated (IB4+ $\mathrm{N}=48$; CGRP, $\mathrm{N}=58$; t-test, $\mathrm{P}<0.001$ ) and STZ-treated mice (IB4+ $\mathrm{N}=57$; CGRP, $\mathrm{N}=56$; t-test, $\mathrm{P}<0.001)$. Abbreviations: IB4: isolectin B4; CGRP: calcitonin gene-related peptide; CTR: vehicle-treated mice; STZ: streptozotocin-treated mice. ${ }^{* * * *} \mathrm{P}<0.0001$.

Fig. 2 Analysis of the changes in the number of SGCs induced by STZ. (A-D) Representative pictures of CGRP (blue), IB4 (green) and DAPI (white) staining on whole-mount DRGs. (E) Histograms illustrating the number of DAPI+ nuclei surrounding CGRP+ or IB4+ sensory neurons normalized to the cross-sectional area in vehicle- and STZ- treated mice. Two-way ANOVA: effect of treatment: $F(1,216)=0.02, P=0.89$; effect of the cell phenotype: $F(1,216)$ $=84.89, \mathrm{P}<0.001$; interaction between treatment and phenotype: $\mathrm{F}(1,216)=0.48, \mathrm{P}=0.16$. $\mathrm{T}$ Test: IB4-CTR vs CGRP-CTR, IB4-CTR vs CGRP-STZ, IB4-STZ vs CGRP-CTR, IB4-STZ vs CGRPSTZ, P<0.001; IB4-CTR vs IB4-STZ, CGRP-CTR vs CGRP-STZ, P > 0.05). Abbreviations: IB4: 
isolectin B4; CGRP: calcitonin gene-related peptide; DAPI, 4',6-diamidino-2-phenylindole; CTR: vehicle-treated mice; STZ: streptozotocin-treated mice. ${ }^{* * * *} \mathrm{P}<0.0001$.

Fig. 3 GS immunostaining and analysis of SGC coverage of CGRP+ and IB4+ neurons. (A-B) Representative images showing double staining for the SGC marker GS (red), the peptidergic DRG neuron marker CGRP (blue, A), and the non-peptidergic DRG neuron marker IB4 (green, B) in vehicle-treated and STZ-treated mice. (C) Method for GS staining analysis. On the left, representative single optical section of GS staining around a DRG neuron. Fluorescence intensity is measured along the four colored lines, which cross the ensheathing SGC in 8-points (white dots) around the DRG neuron. Quantification of the fluorescence intensity along each colored line is illustrated in the graph on the right, using the same color code. GS fluorescence intensity is measured at the white dots, i.e. the peaks in the graphs, and then normalized to internal background (yellow dot). (D) Minimal, mean and maximal GS fluorescence intensities around CGRP+ or IB4+ neurons vehicle-treated mice. T-test: Min, $\mathrm{P}=0.053$; Mean, $\mathrm{P}=0.028$; Max, $P=0.079$. (E) Minimal, mean and maximal GS fluorescence intensities around CGRP+ or IB4+ neurons in STZ-treated mice. T-test: Min, $\mathrm{P}=0.016$; Mean, $\mathrm{P}=0.028$; $\mathrm{Max}, \mathrm{P}=0.056$. (F) Dot plot graph of minimal GS fluorescence intensity. Two-way ANOVA: interaction between treatment and phenotype: $\mathrm{F}(1,218)=9.49, \mathrm{P}=0.002$; effect of treatment: $\mathrm{F}(1,218)=2.99, \mathrm{P}=$ 0.085; effect of the cell phenotype: $\mathrm{F}(1,218)=0.02, \mathrm{P}=0.88$. IB4-CTR vs IB4-STZ, $\mathrm{t}$-test, $\mathrm{P}=$ 0.0014; CGRP-CTR vs CGRP-STZ, t-test, $P=0.32$. (G) Dot plot graph of mean GS fluorescence intensity. Two-way ANOVA: interaction between treatment and phenotype: $F(1,218)=9.26, P$ $=0.003$; effect of treatment: $F(1,218)=8.45, P=0.36$; effect of the cell phenotype: $F(1,218)=$ $0.015, \mathrm{P}=0.9$. IB4-CTR vs IB4-STZ, t-test, $\mathrm{P}=0.005$; CGRP-CTR vs CGRP-STZ, t-test, $\mathrm{P}=0.14$. $(\mathrm{H})$ Dot plot graph of maximal GS fluorescence intensity. Two-way ANOVA: interaction between treatment and phenotype: $\mathrm{F}(1,218)=6.12, \mathrm{P}=0.014$; effect of treatment: $\mathrm{F}(1,218)=0.17, \mathrm{P}=$ 0.68; effect of the cell phenotype: $\mathrm{F}(1,218)=0.017, \mathrm{P}=0.69$. IB4-CTR vs IB4-STZ, $\mathrm{t}$-test, $\mathrm{P}=$ 0.13; CGRP-CTR vs CGRP-STZ, t-test, $\mathrm{P}=0.048$. Abbreviations: IB4: isolectin B4; CGRP: calcitonin gene-related peptide; CTR: vehicle-treated mice; STZ: streptozotocin-treated mice; GS: glutamine synthetase. ${ }^{*} \mathrm{P}<0.05,{ }^{* *} \mathrm{P}<0.01$.

Fig. 4 Ultrastructure of the DRG neurons in control mice. (A) An ensheathing layer made of SGC processes (arrowheads) separates two adjacent neurons. (B) The neuronal membranes of two adjacent neurons are in direct contact (arrows), without SGC interposition. (C) Highmagnification of panel A. Note the presence of an SGC process between the sensory neuron somata (arrowheads) and gap-junctions between neuron and SGC (red double arrowheads). (D) High magnification of panel B. Note the lack of SGC interposition between the facing membranes of the two neurons (arrows). Abbreviation: SGC: Satellite glial cell; N: nucleus; ct: connective tissue.

Fig. 5 Ultrastructural analysis of IB4+ DRG neurons in control and diabetic mice. (A) In CTR, the membranes of two adjacent clustered IB4+ sensory neurons are juxtaposed, without the interposition of glia (arrowheads). (B) In STZ-treated mice, a glial sheath is present between two IB4+ neurons of the same cluster (arrows). (C) High magnification of panel A. Note the occurrence of $20 \mathrm{~nm}$ gold particles indicative of IB4 immunogold staining scattered over the entire length of the juxtaposed neuronal membranes (arrowheads). (D) High-magnification of panel B. Note the glia separating the membranes of two IB4+ DRG neurons (arrows) and a gapjunction between the neuron and SGC (red double arrowheads). (E) Contact index in vehicletreated mice and STZ-treated mice. The contact index is markedly reduced in STZ (MannWhitney test, $\mathrm{P}<0.01$ ). (F) Pie charts showing the proportion of neuronal membranes exhibiting at least one point of contact (Fisher exact test, $\mathrm{P}<0.05$ ) in CTR and STZ-treated mice. Abbreviation: SGC: Satellite glial cell; N: nucleus; CTR: vehicle-treated mice; STZ: streptozotocin-treated mice. 
Fig. 6 Schematic summary of neuro-glia relationship in DRGs of control and diabetic mice. (A) Representation of a simplified dorsal root ganglion (DRG) in control (CTR) mice: IB4+ neurons (green) are grouped in cluster and surrounded by a few satellite glial cells (SGC). SGCs form a continuous glutamine synthetase (GS)+ sheet around neurons (red thick line) which becomes thinner at the interface of two IB4+ opposing neurons. At some points, the membranes of cluster-forming IB4+ neurons are in direct contact (yellow arrowheads). CGRP+ neurons (blue) are randomly scattered across the DRG and surrounded by numerous SGCs with relatively less bright GS immunostaining (red thin line). (B) In streptozotocin (STZ)-induced diabetic mice, IB4+ neurons (green) are still grouped in clusters and CGRP+ neurons (blue) randomly scattered across the DRG. However, GS fluorescence intensity is higher around CGRP+ neurons (red thick line) than in IB4+ neurons (red thin line). Moreover, the points of the cluster where two IB4+ opposing neurons are in direct contact are significantly reduced.

\section{Supporting Information}

Additional Supporting Information may be found in the online version of this article:

Data S1 Computerized analysis of neuronal clusterization.

Fig. S1 Blood glucose concentration in control and diabetic mice.

Fig. S2 Schematic flowchart describing the steps in image processing performed using the $3 D R G$ software.
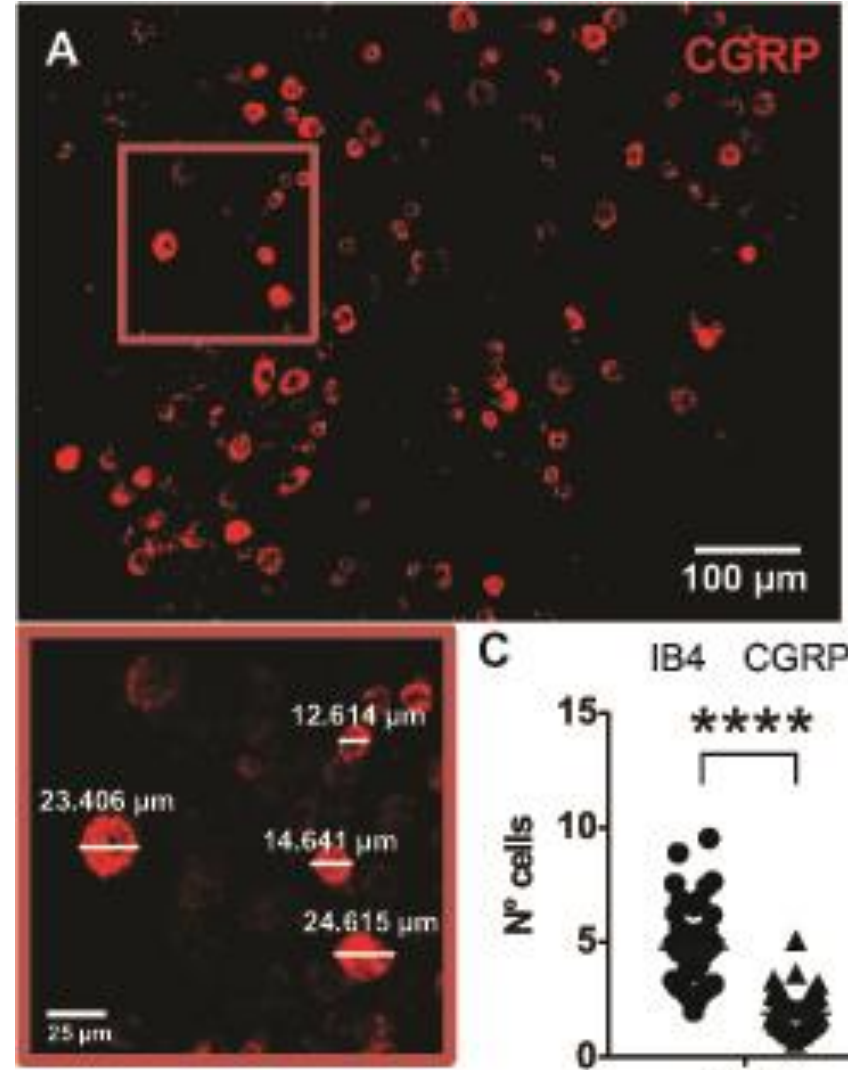

Figure 1

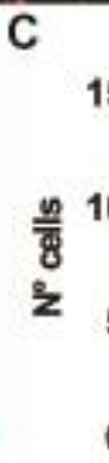

IB4 CGRP

$* * * *$
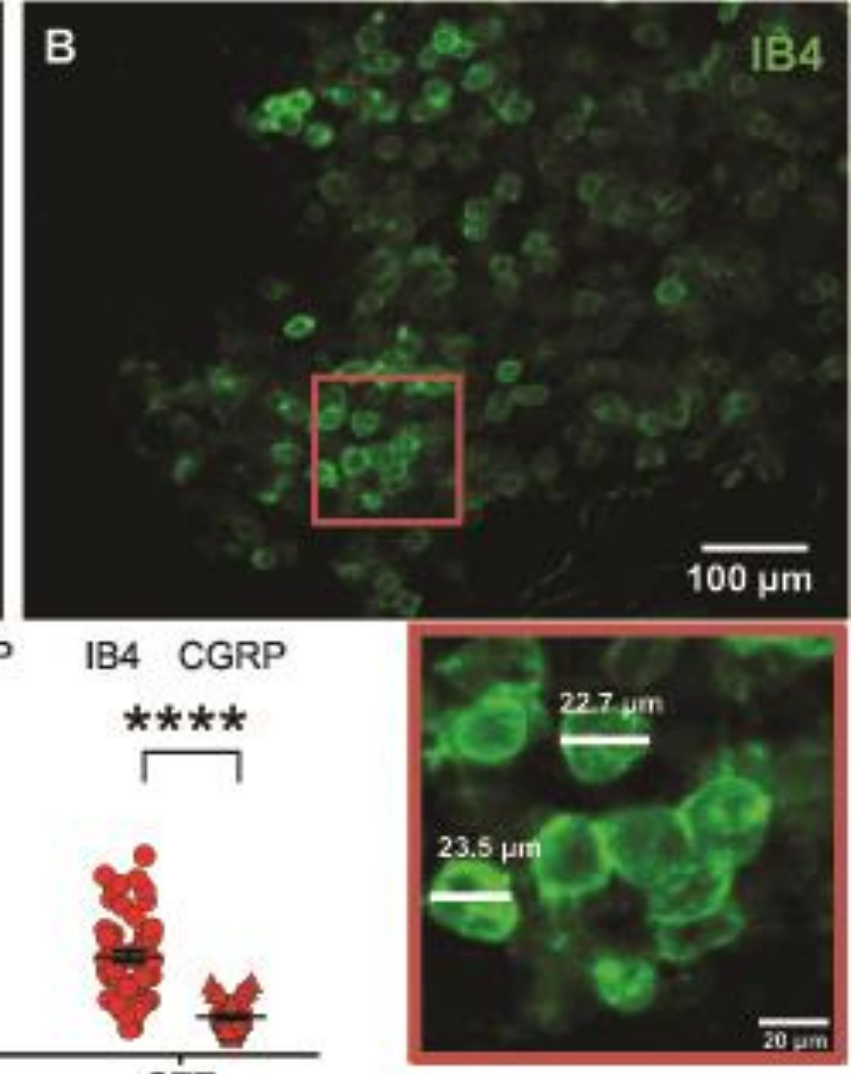

STZ 

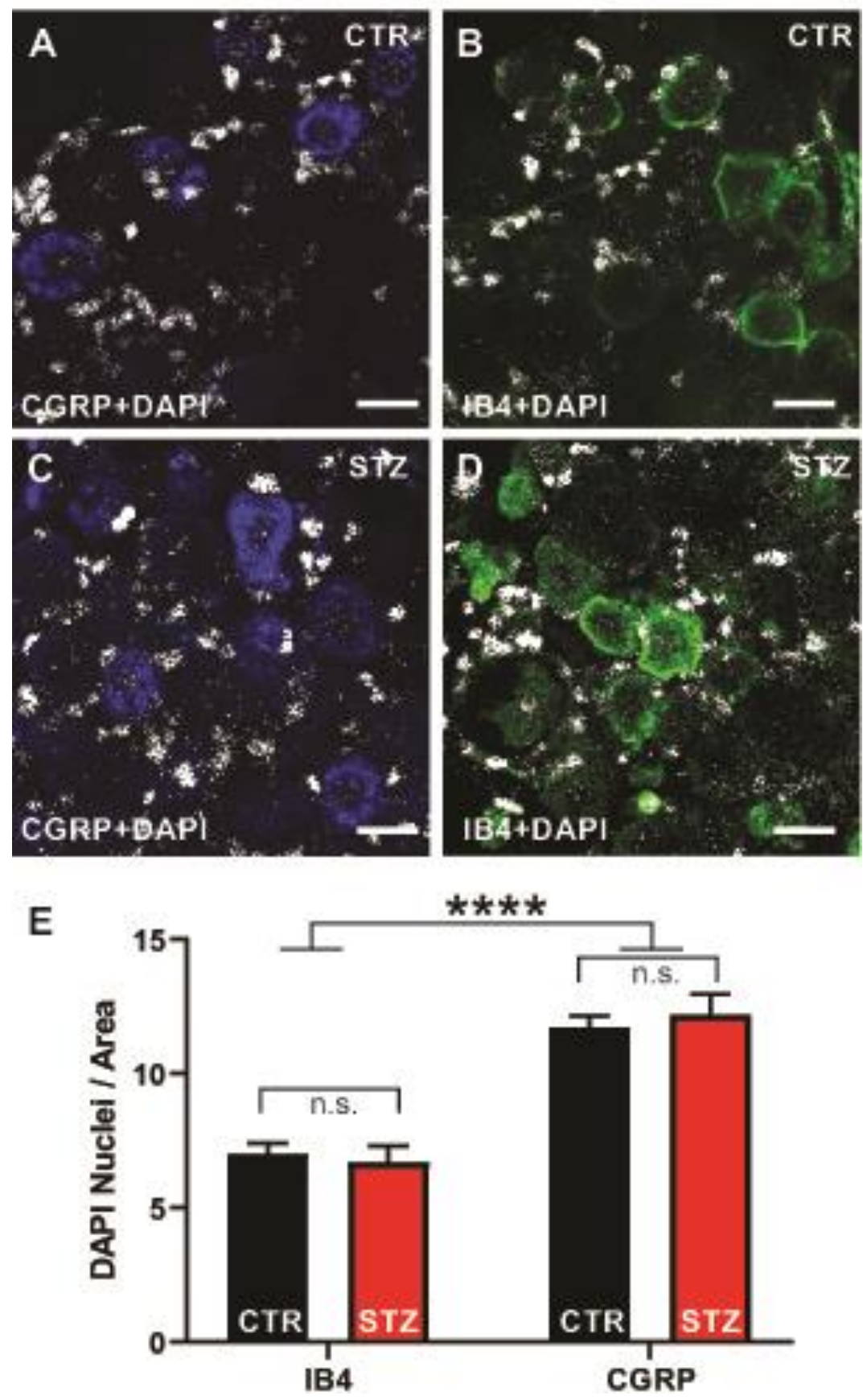

Figure 2 
A

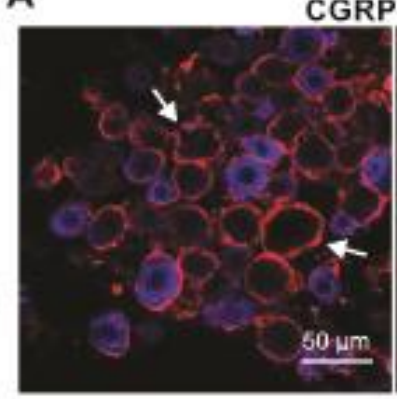

CTR

c

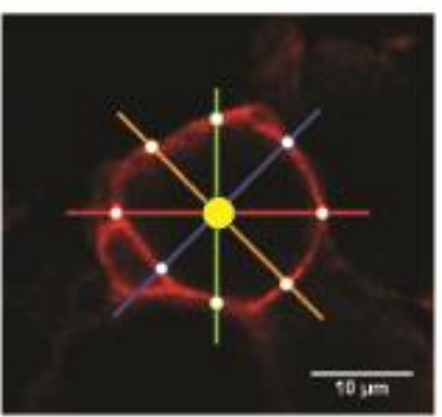

D

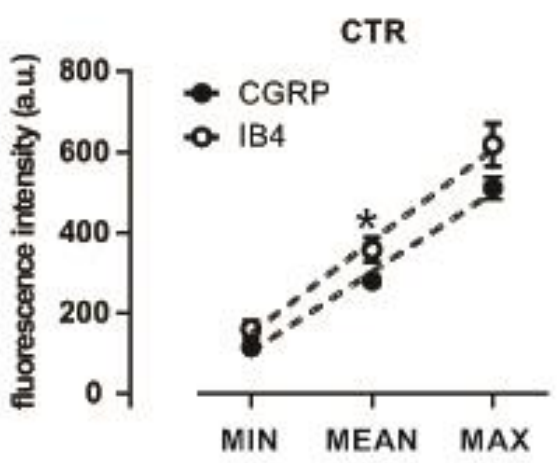

B

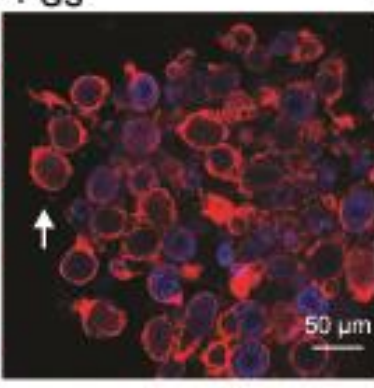

STZ

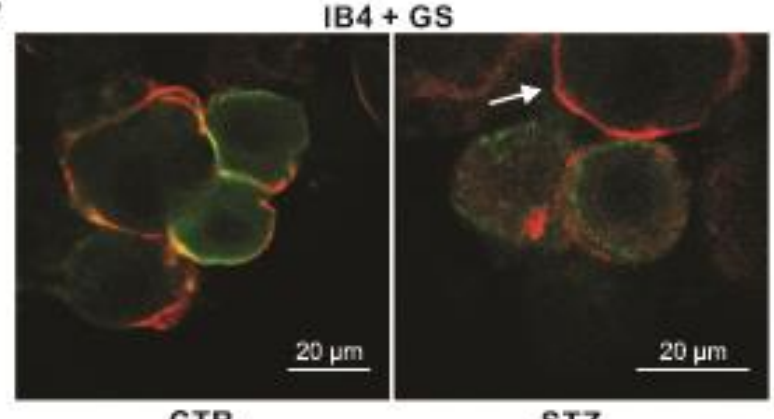

STZ

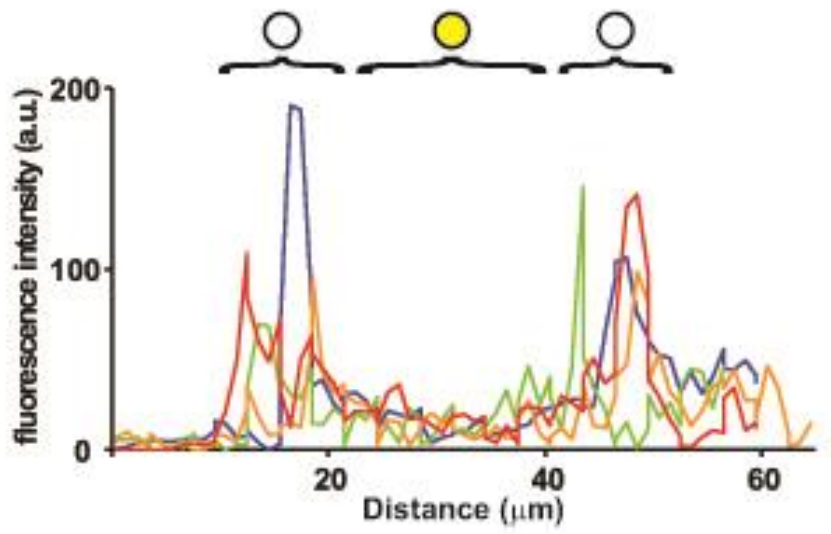

E

STZ

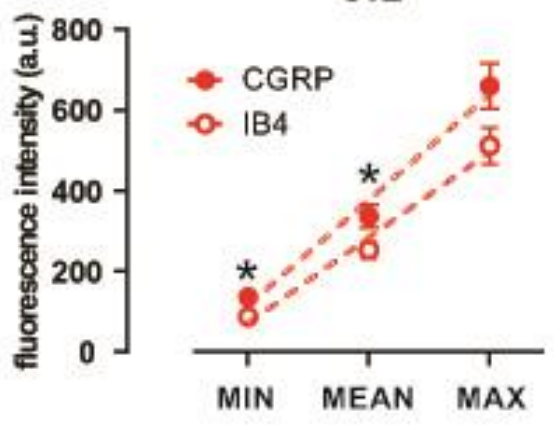

F

G
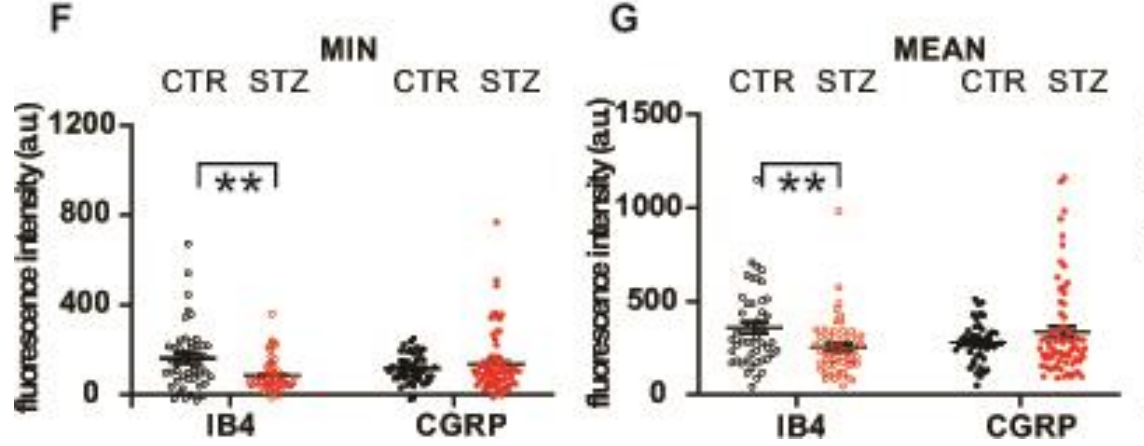

H

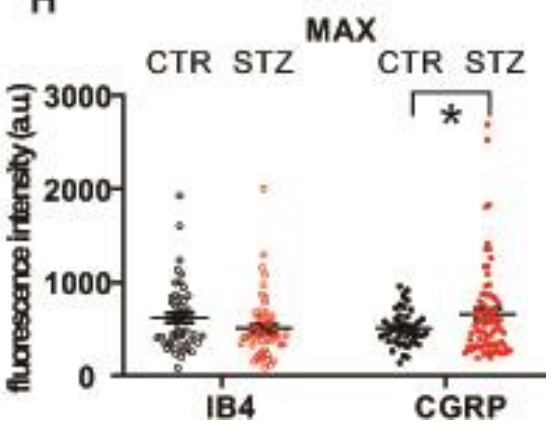

Figure 3 

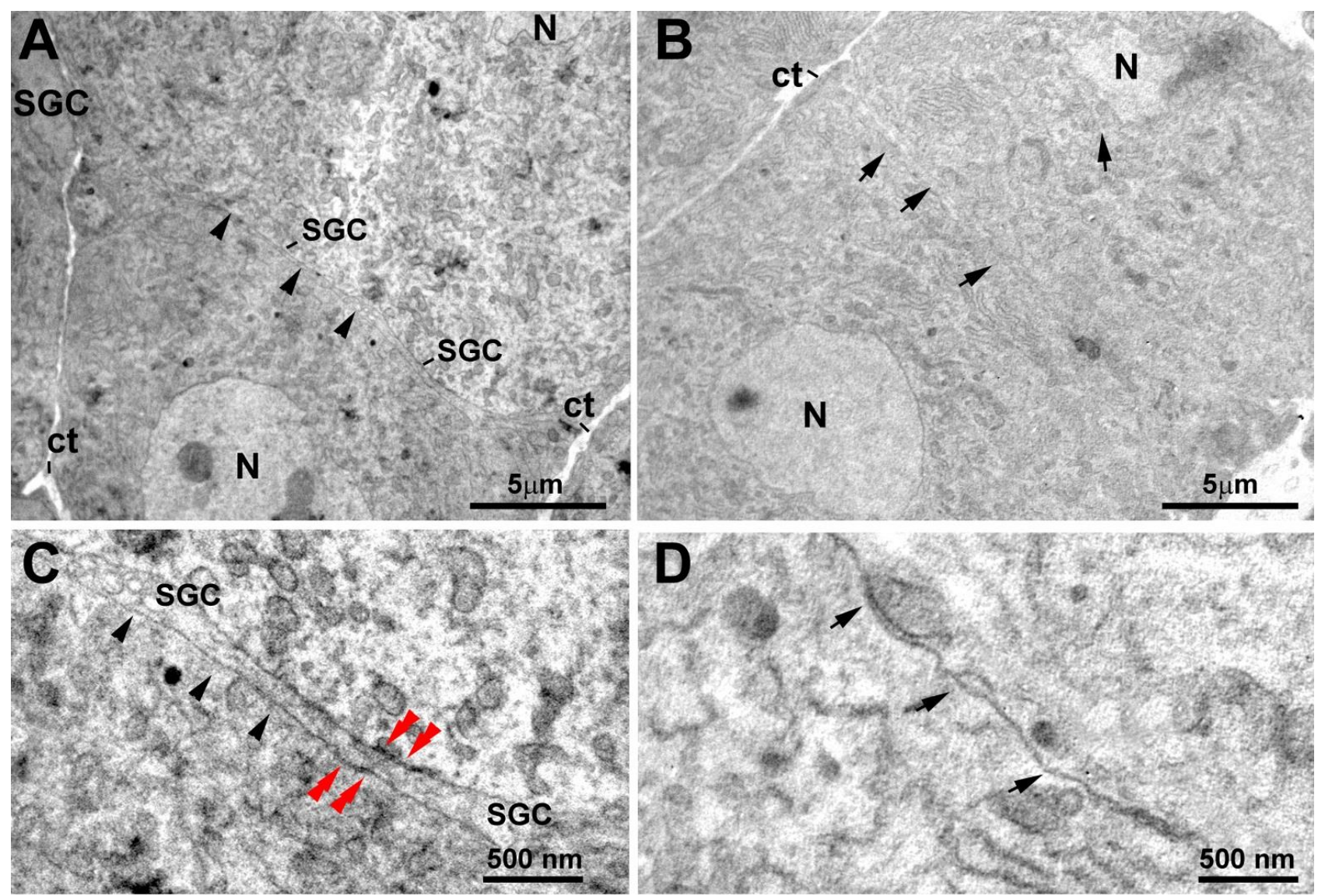

Figure 4 

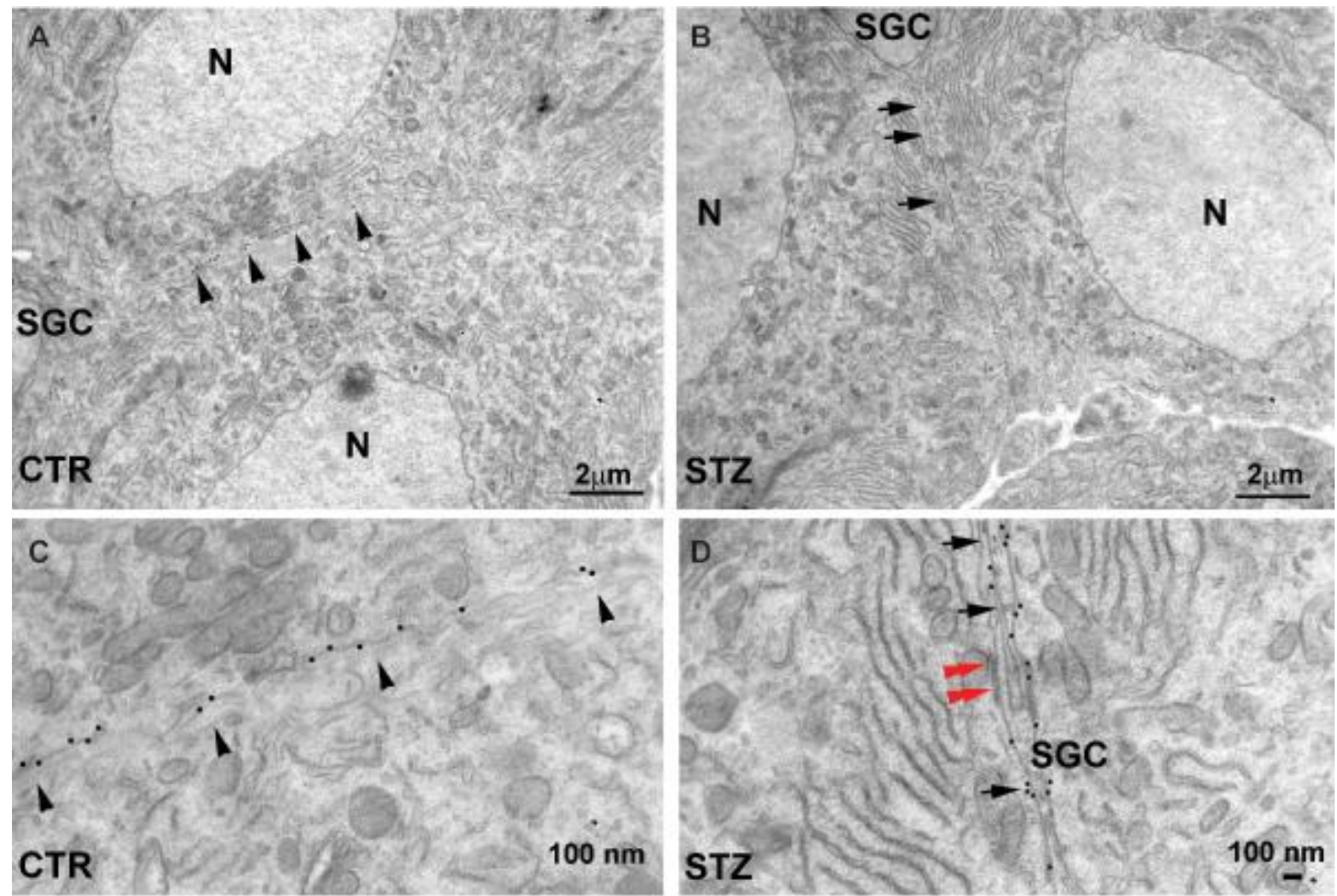

E

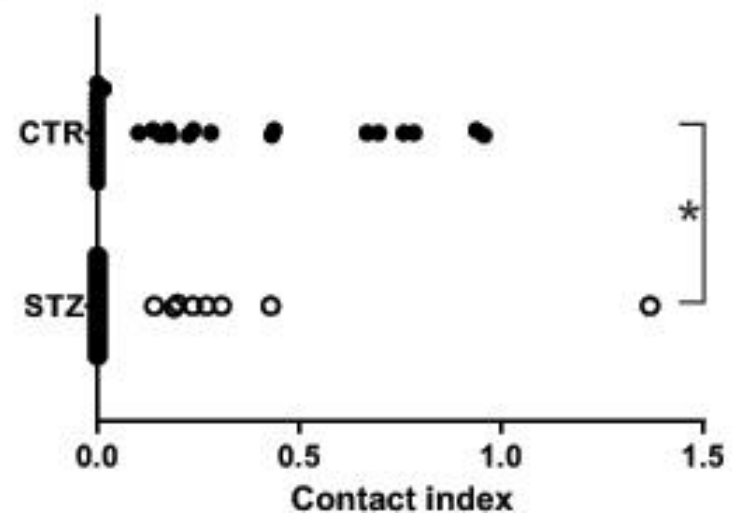

F

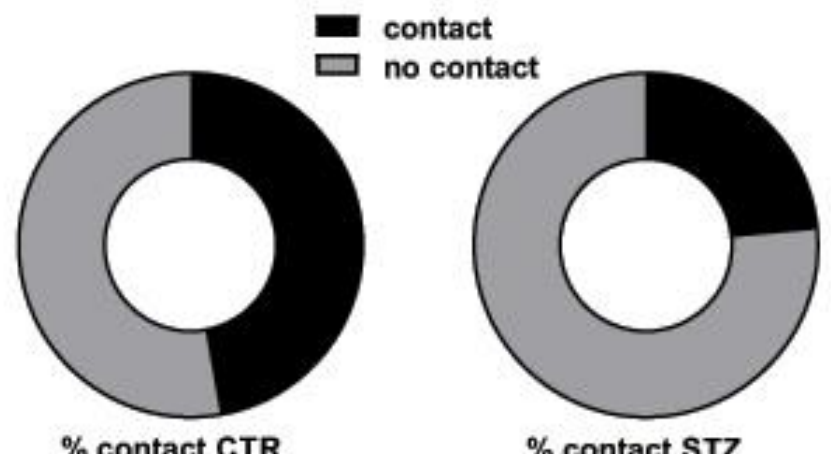

Figure 5 


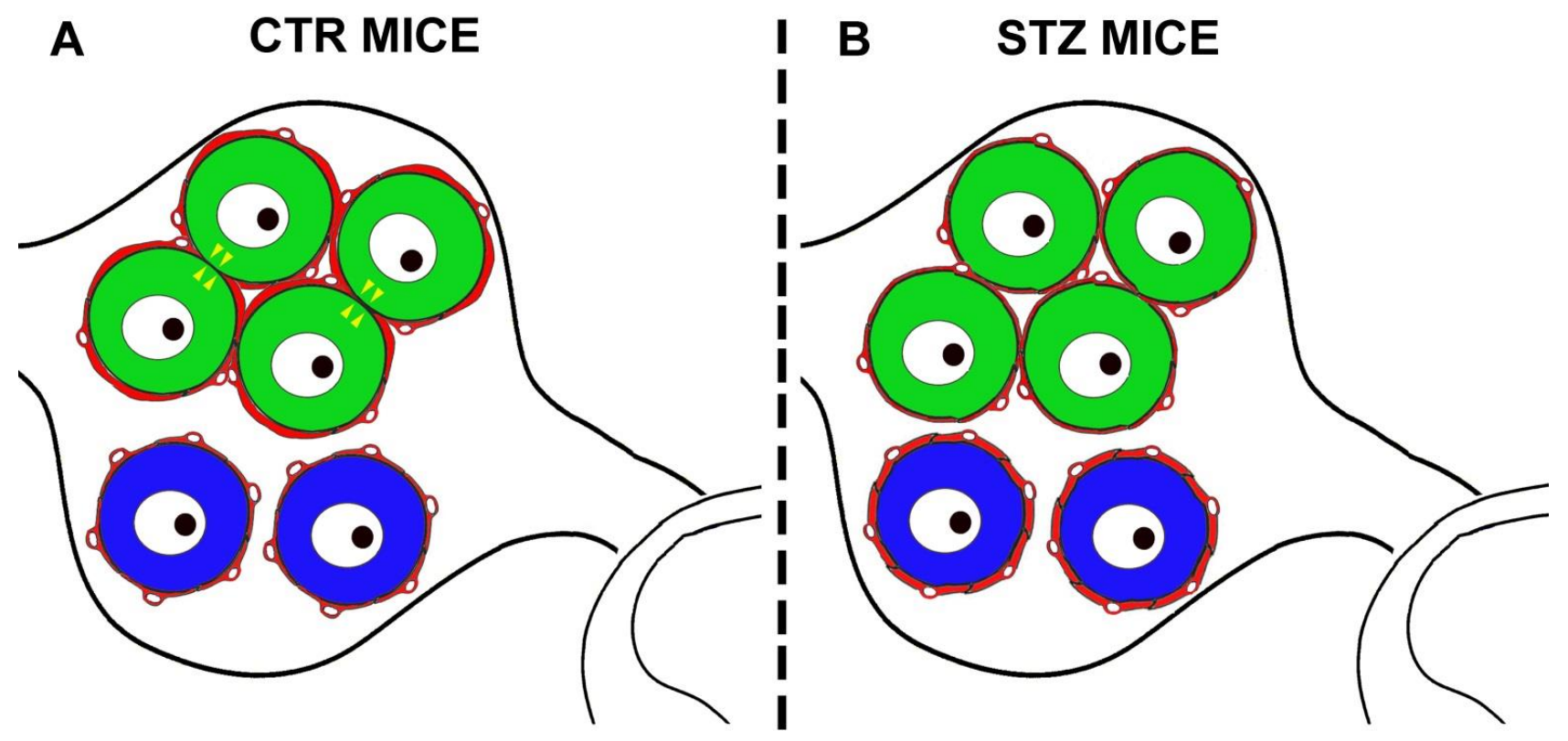

Figure 6

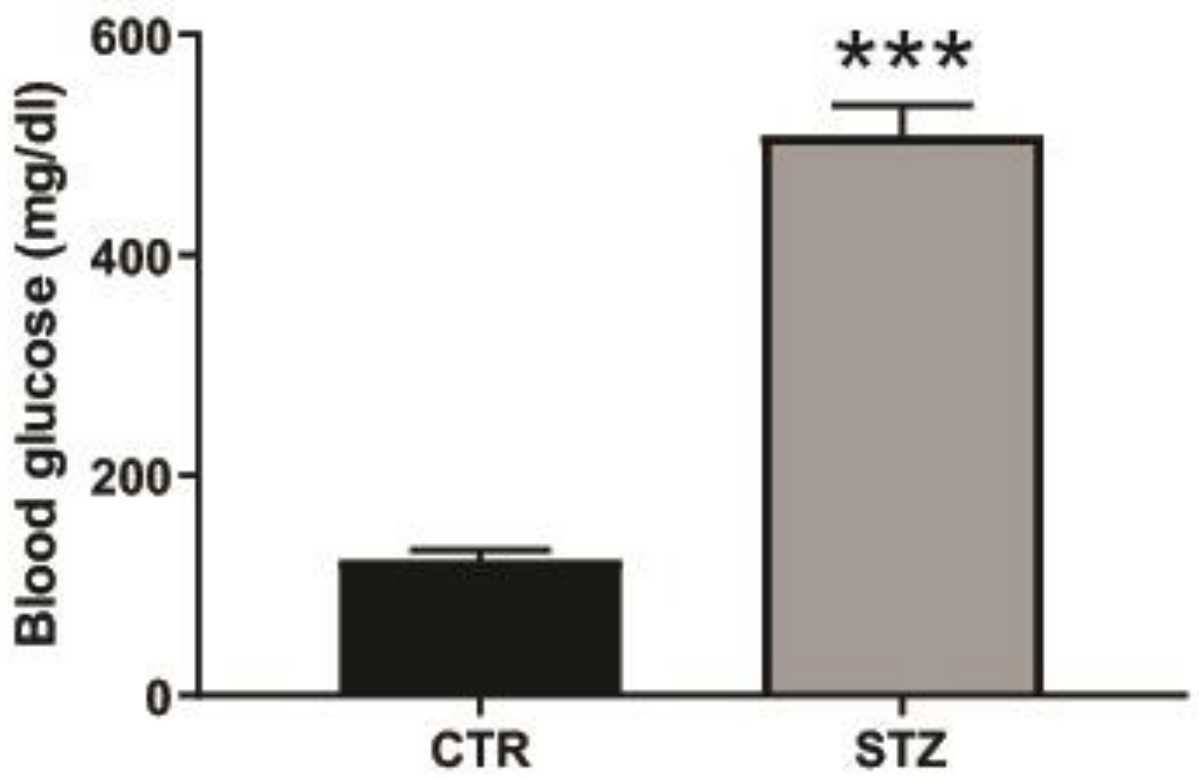

Supplemental Figure 1 
INPUT:

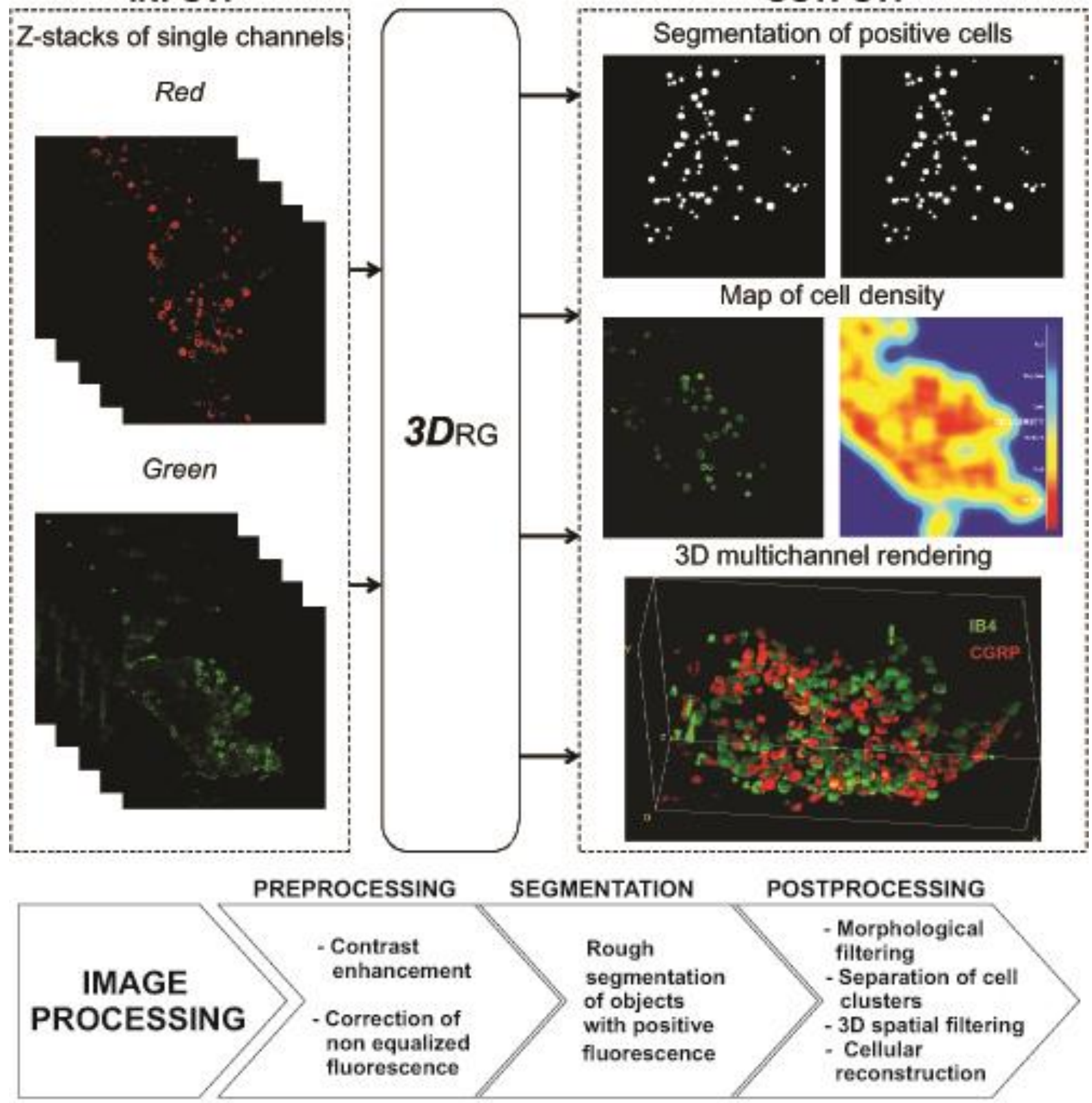

Supplemental Figure 2 